\title{
El aula invertida transforma el proceso de enseñanza-aprendizaje de estudiantes
}

\section{normalistas}

\section{The flipped classroom transforms the teaching learning process of the students from the Normal Rural School}

\author{
María Eliud Reyes Pinzón ${ }^{1}$ \\ José Benito Dzul Tamay² \\ Elias Miguel Melken Balam ${ }^{3}$
}

\section{Resumen}

Hoy en día en nuestro México se vive un cambio se percibe una creciente y preocupante falta de motivación, esfuerzo, comprensión y aprendizaje por parte de los jóvenes por aprender, el docente del siglo XXI requiere de innovadoras técnicas para apoyar su clase. Se plantea la necesidad de innovar la metodología tradicional por una que aproveche los diferentes recursos tecnológicos para incorporarlos en forma efectiva en su práctica docente y enseñanza. El presente estudio analiza la metodología Aula Invertida mediante el uso de las TIC y su influencia en el aprendizaje de los estudiantes

\footnotetext{
1 María Eliud Reyes Pinzón. Docente en la Escuela Normal Rural Justo Sierra Méndez, Hecelchakan, Campeche, Mexico. Es doctora en educación y actualmente forma parte de la Red Temática de investigación de Educación Rural. Correo electrónico: marielyta_reyes@hotmail.com

ID: http://orcid.org/0000-0002-2581-8111
}

2 José Benito Dzul Tamay. Docente en la Escuela Normal Rural Justo Sierra Méndez, Hecelchakan, Campeche, Mexico. Impare los cursos del trayecto formativo de Práctica Profesional y el de las lenguas y culturas de los pueblos originarios. Correo electrónico: dzultamayjb21@hotmail.com

ID:http://orcid.org/0000-0002-8766-2001

3 Elias Miguel Melken Balam. Docente en la Escuela Normal Rural Justo Sierra Méndez, Hecelchakan, Campeche, Mexico. Maneja la línea de investigación sobre innovación educativa desde la perspectiva del diseño curricular y los procesos pedagógicos y didácticos. Correo electrónico: melken30@hotmail.com ID: http://orcid.org/0000-0002-0862-3638 
de la Escuela Normal Rural de Hecelchakán, el objetivo principal de este trabajo es la implementación en un grupo piloto la técnica de aula invertida para mejorar los resultados del proceso enseñanza y aprendizaje. Se aplicó un programa piloto a un solo grupo con un total de 25 alumnos el trabajo se diseñó con la investigación de pre-prueba y pos-prueba, finalmente se presentan los resultados, conclusiones y recomendaciones.

\title{
Palabras clave
}

Aula invertida, aprendizaje colaborativo, TIC, enseñanza-aprendizaje.

\begin{abstract}
Nowadays in our Mexico we are experiencing a change. We perceive a growing and worrying lack of motivation, effort, understanding and learning on the part of young people to learn, the 21st century teacher requires innovative techniques to support their class. There is a need to innovate the traditional methodology by one that takes advantage of the different technological resources to incorporate them effectively in their teaching and teaching practice. The present study analyzes the Inverted Classroom methodology through the use of TIC and its influence on the learning of the students of the Rural Normal School of Hecelchakán, the main objective of this work is the implementation in a pilot group the inverted classroom technique for improve the results of the teaching and learning process. A pilot program was applied to a single group with a total of 25 students. The work was designed with pre-test and post-test research, finally the results, conclusions and recommendations are presented.
\end{abstract}

\section{Keywords}

Flipped classroom, collaborative learning, TIC, teaching-learning.

\section{Planteamiento del problema}

Vivimos en la sociedad de la información, atentos a los cambios tecnológicos y económicos del desarrollo, a nivel mundial se observa el enorme avance de los recursos informáticos como son las Tecnologías de Información y Comunicación que impulsan cambios en la educación. Si los alumnos se encuentran en una sociedad donde el desarrollo tecnológico y sus aplicaciones es una realidad que cada día está presente en sus vidas, se les tendrá que dotar de competencias con el uso de TIC. En este escenario se plantea la necesidad creciente que los docentes estén en condiciones de cambiar su metodología tradicional de enseñanza por un nuevo modelo 
llamado aula invertida metodología que se basa en las mismas ideas, esta realidad, nos lleva a la siguiente pregunta de investigación:

¿Es posible que el aula invertida mejore el proceso enseñanzaaprendizaje de los normalistas del $1^{\circ}$ grupo "A" de la Escuela Normal Rural "Justo Sierra Méndez" mediante el apoyo de TIC?

La hipótesis plantea que existe una relación positiva entre la metodología Aula invertida mediante TIC y el proceso enseñanza aprendizaje en los alumnos del $1^{\circ}$ grupo " $A$ " de la Escuela Normal Rural "Justo Sierra Méndez".

Delimitación espacial: se realizarán por dos medios o espacios.

- El primero, se realizó un aula virtual MOODLE del curso Las TIC en la educación para el grupo $1^{\circ} \mathrm{A}$.

- Segundo se trabajó en el aula física $1^{\circ}$ grupo B de la escuela normal rural justo sierra Méndez.

- Delimitación temporal: Se aplicó la metodología del aula invertida en los meses de agosto 2016 a febrero 2017.

- Delimitación práctica La intención es aplicar la metodología del aula invertida que es una novedosa metodología la cual apoyará en primer lugar a los estudiantes y a la docente de la escuela normal rural

\section{Marco teórico}

(Calvillo 2014, p 8-9) Según la tesis “Flipped Learning” aplicado a la materia de música en el cuarto curso de Educación Secundaria Obligatoria: una investigación-acción para la mejora de la práctica docente y del rendimiento académico del alumnado, nos quiere dar a conocer que el modelo metodológico conocido -en la mayoría de los círculos docentes- como Flipped Classroom (FC) o "clase del revés", es un fenómeno que está recibiendo un considerable incremento de atención en círculos dedicados a la educación, así como en prensa especializada (Toppo, 2011; Tucker, 2012). En este modelo, el alumnado recibe la parte instruccional de la enseñanza en casa a través de vídeos y otros medios multimedia e interactivos, y las horas de clase, se dedican a resolver dudas sobre las tareas encomendadas, discutir a fondo sobre aquello que más les cuesta comprender o trabajar en problemas y proyectos, etc. La resolución de problemas en grupos, las metodologías colaborativas y cooperativas en clase o las basadas en proyectos.

En definitiva, la enseñanza centrada en el alumnado, la clase pasa de ser de recepción pasiva a ser de participación activa. 
García (2015) en su trabajo "El aula invertida y otras estrategias con uso de TIC. Experiencia de aprendizaje con docentes" describe una experiencia de aplicación diferenciada de estrategias de enseñanza aprendizaje con apoyo de Tecnologías de la Información y la Comunicación (TIC) en una comunidad de docentes que son estudiantes de la Maestría en Educación.

Carneiro (2008) -por su parte- señala en "Los desafíos de las TIC para el cambio educativo", el tema de el aula invertida y otras estrategias con uso de TIC. Experiencia de aprendizaje con docentes, se desprende de un proyecto que se inició en la Universidad Interamericana para el Desarrollo (UNID), en la Sede Tuxtepec, Oaxaca, México, con alumnos de la Maestría en Educación, que, a su vez, se desempeñan como docentes en la zona. En esta investigación se busca identificar la posibilidad de mejoras en los procesos educativos.

\section{Metodología}

La metodología son los pasos que se deben de seguir para llevar a cabo la investigación en este caso. El diseño, con prueba-pos prueba y grupo de control: A un grupo se le aplica una prueba previa al estímulo o tratamiento experimental. Después se le administra el tratamiento y para terminar se le aplica una prueba posterior al tratamiento, hay un punto de referencia inicial para ver qué nivel tenía el grupo en las variables dependientes ante el estímulo. Es decir, hay un seguimiento del grupo. Este diseño se aplicará de la siguiente manera:

Población y muestra.

- Población: Estudiantes normalistas de la escuela normal rural "Justo Sierra Méndez"

- Muestra: 25 estudiantes del $2^{\circ}$ grupo A de la asignatura de las TIC en la educación matriculados en el semestre 2016.

Técnicas e instrumento de recolección de datos.

Precisamos encontrar los medios de recolección de datos para luego procesarlas, analizarlas y posteriormente extraer las conclusiones sobre la base de la información recolectada, aplicando las técnicas e instrumentos pertinentes como: Encuestas y Guías de Observación.

\section{Procesamiento de datos}

Para el entendimiento e interpretación de los resultados en estudio se realizará el procesamiento de datos con el programa de SPSS o Microsoft 
Excel donde se tabulará y se verá las frecuencias sobre las preguntas hechas en las encuestas realizadas a los alumnos.

La comprobación de datos tablas, gráficas y productos se encuentran en el portafolio de evidencias en línea de este trabajo es explicativodescriptivo.

\section{Desarrollo}

La técnica del aula invertida puede ser entendida como "dar la vuelta a la clase" o "una clase al revés". Este nuevo término sirve para definir un nuevo método docente cuya base radica en la metodología del "aula invertida" las tareas que antes se hacían en casa, ahora se realizan en clase y a la inversa.

Los primeros autores que acuñaron el término fueron Lage, Platt, y Treglia (2000). Aunque realmente la expresión no se consolidó hasta que en 2007 los profesores Bergman y Sams, del Instituto de Colorado de USA, se unen para grabar contenidos de presentaciones en PowerPoint narradas y capturadas en vídeo. El objetivo era facilitar a los alumnos que no pudieran asistir a clase la posibilidad de seguir si no toda, gran parte de la materia a través de las grabaciones que realizaban los profesores.

Para sorpresa de los docentes, estas grabaciones, en principio creadas para estudiantes no presenciales de sus clases, eran también seguidas por otros estudiantes.

El potencial de esta metodología docente radica en que el tiempo invertido en explicar el curso, por ejemplo, a través de la clase magistral, queda relegado al trabajo que el alumno puede hacer tranquilamente en casa a través de grabaciones en un vídeo you-tube o en una presentación narrada en Power Point. Estos materiales pueden ser visionados y estudiados por los alumnos en su propio domicilio, con la ventaja de que pueden hacerlo cuantas veces considere necesario.

De este modo, existe una complementación entre la técnica del aula invertida y el aprendizaje cooperativo: las tareas, también comúnmente conocidas como "deberes" se realizan conjuntamente y en cooperación con el grupo ya que, el docente traslada el tiempo empleado a la explicación de la materia al método aula invertida, o tarea en casa.

El objetivo es implementar un grupo piloto con la técnica de aula invertida para mejorar los resultados del proceso enseñanza y aprendizaje con la creación de ambientes de colaboración donde se desenvuelvan los educandos.

Durante el desarrollo del curso Las Tic en la Educación se aplicó un pilotaje de innovación y mejora docente. Para la presentación de este trabajo, se realizó una investigación no experimental, de tipo descriptivo, transversal 
RECIE. Revista Electrónica Científica de Investigación Educativa Vol. 4, núm. 2, enero-diciembre 2019, pp. 1247-1258.

(Cea, 2009; Hernández, Fernández-Collado y Baptista, 2014). El grupo piloto estuvo formado por 25 alumnos del primer grado grupo A, de los cuales son 12 hombres y 13 mujeres cuyas edades oscilan entre 18-21 años, el curso se impartió en el primer grado (Agosto-2016/Enero-2017) los alumnos estuvieron asignados en cuatro equipos de trabajo.

Las competencias del perfil de egreso a las que contribuye el curso son las siguientes:

- Usa las TIC como herramienta de enseñanza y aprendizaje.

- Actúa de manera ética ante la diversidad de situaciones que se presentan en la práctica profesional.

- Utiliza recursos de la investigación educativa para enriquecer la práctica docente, expresando su interés por la ciencia y la propia investigación.

- Las competencias del curso son:

- Utiliza de manera crítica y creativa las herramientas de productividad capaces de solucionar problemas.

- Usa las TIC como herramienta para publicar, comunicar, colaborar y producir información de calidad que contribuya a la sociedad.

- Actúa de manera ética ante el tratamiento de la información.

- Los contenidos docentes de la asignatura estuvieron expuestos en la plataforma virtual del curso para que los alumnos pudieran tener acceso a ellos desde cualquier punto con acceso a internet. Esta plataforma es una herramienta de apoyo a la docencia y desde donde los estudiantes pueden acceder e intercambiar información.

A los estudiantes se les informó al inicio del curso, sobre el proyecto piloto de innovación y mejora docente, fomentando en todo momento la comunicación, con los alumnos, sincrónica como asincrónica, para mantener una relación alumnado-profesorado más interactiva, y sin contar con la barrera de la presencialidad, como sucede en las tutorías establecidas en unos horarios, que muchas veces, son coincidentes con otras actividades docentes para los alumnos, como puedan ser las clases, seminarios o las prácticas.

Para la recolección de datos se diseñó un cuestionario de opinión que se les aplico a los estudiantes al finalizar el semestre, para valorar la experiencia y satisfacción de los estudiantes con el Aula Invertida.

Se concentraron datos sociodemográficos: 1. Sexo. 2. Edad. Se consultaron tres preguntas sobre el uso del Aula Invertida, con respuestas tipo Likert, estas fueron: 1. Me facilita el estudio previo. 2. Mejoro mi rendimiento académico. 3. Grado de satisfacción. Además, se preguntaron 
dos cuestiones abiertas sobre el uso del Aula Invertida: 1. Aspectos positivos. 2. Aspectos negativos.

En cuanto a las normas que se siguieron durante el curso, estas fueron las siguientes:

El curso se fundamentó en el trabajo en equipo y colaborativo, fomentando las búsquedas de información autónomas. En cada equipo se elaboraron las normas necesarias para una buena integración de todos los miembros en el mismo. Se establecen las normas en cada equipo de trabajo y éstas se deben seguir, a partir de entonces, con el resto de los integrantes del equipo.

Los estudiantes tenían que asistir a la clase, que requirieran búsquedas de información, con los portátiles para tener acceso a internet, preparar los informes. Si alguno/a no disponía de portátil, debía cerciorarse de incorporarse a un equipo, donde el resto de los compañeros dispongan de los mismos.

En cada equipo de trabajo se tuvo que nombrar un/a moderador/a y secretario/a. El primero/a se encargó de que todos los miembros del mismo participaran, en la medida de lo posible, por igual. El segundo/a se encargó de exponer las conclusiones de los trabajos realizado por cada equipo

La sistematización de datos se realizó en el aula. La participación fue voluntaria y anónima, solicitando previamente el asentimiento informado.

Después de cada unidad vista en el curso se debía entregar un informe de la actividad realizada en el equipo de trabajo. La persona encargada de entregarlo a la profesora era el secretario de cada equipo.

En cada informe de unidad se tenía que poner al principio del mismo, los nombres y apellidos de cada uno de los integrantes del equipo de trabajo. Los informes y las bibliografías utilizadas en cada unidad se tenían que presentar siguiendo las normas APA de la $6^{\underline{a}}$ edición (American Psychologycal Association, 2010).

En el curso se solicitó a los equipos que trajeran realizadas las búsquedas de información u otro tipo de trabajo, como podía ser lectura de artículo, enlaces páginas web o visionado de vídeos. Esta actividad estaba contemplada en el Proyecto Docente del curso Las Tic en la Educación (curso 2016/2017), dentro del laboratorio de cómputo en horario no presencial de la asignatura.

La maestra del curso llevó a cabo una valoración de cada una de las unidades, mediante una rúbrica de evaluación, en la cual, se presentan los siguientes aspectos:

- Asistencia: Es obligatoria la asistencia. Si algún estudiante necesitaba un cambio puntual por un motivo justificado, tiene que comunicar a la 
profesora para que pudiera gestionar con tiempo, el cambio a otro día y/o centro docente dentro de la misma semana.

- Puntualidad: Se toma en cuenta la puntualidad de la asistencia a cada clase y la puntualidad en la entrega de los informes de cada equipo de trabajo.

- Calidad del trabajo: Se valoraron las fuentes de información utilizadas, teniendo que estar relacionadas con la temática de cada unidad, estar actualizadas (Recomendable de los últimos cinco años) y referenciadas las bibliografías utilizadas según APA 6 ${ }^{\mathrm{a}}$ ed.

- Contribución: Se estimó el logro de objetivos de cada unidad y las búsquedas de soluciones llevadas a cabo en cada equipo de trabajo.

- Normas: Los integrantes de cada equipo de trabajo tuvieron que seguir unas normas de convivencia basadas en el respeto mutuo y debían cumplirlas. En el equipo donde algún miembro no siguiera estas normas, la persona que ostentaba la figura de secretario, debía ponerlo en conocimiento del profesor, con la mayor prontitud.

- Exposiciones de los trabajos: Se tiene en cuenta el componente verbal, como el no verbal, además, de la gestión del tiempo acorde con el tiempo asignado por la profesora

- Actitud: Se valoró que los integrantes de los equipos estuvieran receptivos a aceptar sugerencias y a escuchar las opiniones de la profesora y del resto de compañeros/as.

- Participación: Se fomentó la participación de todos los miembros del equipo de trabajo. En el equipo donde algún miembro no participara, la persona que ostentaba la figura del moderador, debía ponerlo en conocimiento del profesor.

\section{Pasos para implementar el aula invertida}

1. Identifica el escenario.

2. Define la actividad.

3. Consolida el aprendizaje.

4. Evalúa

\section{Discusiones}

Al ser las TIC una herramienta del proceso de enseñanza y aprendizaje, se debería aplicar en la enseñanza diaria de los estudiantes no sólo en el curso de TIC en la educación sino también en todos los cursos porque favorecerán el desarrollo de las competencias mediante el trabajo individual y colaborativo, las TIC fomentara y ayudara la autonomía del normalista, 
promoviendo la competencia digital, y a través del trabajo colaborativo desarrollara las habilidades de los alumnos para organizarse, planificarse e intercambiar opiniones o tratar la información, entre otras.

Hay que motivar a los docentes brindando más herramientas y materiales que los alumnos puedan revisar y puedan apoyarse en su educación donde habrá más tiempo para resolver dudas y consolidar conocimientos en clase. Al asumir los estudiantes la revisión de los materiales desde casa (mediante un video, una lectura, recursos interactivos, etc.), el tiempo de clase puede dedicarse a resolver las dudas, solucionar las dificultades de comprensión o aprendizaje, y trabajar los contenidos de manera individual y colaborativa que beneficiara a los alumnos en su apoyo.

Al usar el aula invertida los maestros tienen que estar en continua motivación hacia los estudiantes. hay que prestar atención más en los alumnos de bajo rendimiento y estar constantemente trabajando con ellos y redescubrir el proceso de aprendizaje como algo divertido, donde son ellos los que asumen responsabilidades, toman decisiones, participan y trabajan mano a mano con sus compañeros para alcanzar objetivos comunes. Aprendiendo, haciendo y no memorizando.

Se recomienda para futuros trabajos diseñar y producir diferentes tipos de recursos TIC alternativos al video para evaluar su impacto como parte de la metodología Aula invertida.

\section{Resultados}

Un total del 75\% de los alumnos han afirmado estar, satisfechos con el Aula Invertida porque les facilita el estudio y mejora el rendimiento académico. Los estudiantes han realizado más comentarios positivos sobre el Aula Invertida, que negativos. Los positivos han estado relacionados con la mejora de la relación profesorado-alumnado, del trabajo en equipo y del rendimiento académico.

Se presentó la teoría correspondiente para incluir la metodología aula invertida mediante TIC en el aprendizaje de los alumnos. Según la clasificación de (Monge J y Pérez A, 2008) la prueba de hipótesis para las variables cuya muestra son los estudiantes del curso, se empleará la prueba $\mathrm{T}$ de Student "Estamos interesados en determinar si dos variables una referida a una metodología y la otra en individuos de una población están relacionadas. Se diferencia de los contrastes anteriores en que en este caso estamos interesados en calcular las diferencias existentes entre cada observación del grupo y la observación asociada en el segundo (T de Student para muestras relacionadas), así poder observar que existen relación entre las dos variables de una misma población.

El aula invertida transforma el proceso de enseñanza-aprendizaje... 
Hipótesis de investigación 1: El promedio de las calificaciones de estudiantes entre una clase tradicional es de 7.8 y en la que se aplica la metodología Aula invertida mediante el uso de TIC fue de 8.9.

Hipótesis estadística 1:

- H1: Existe una diferencia significativa del promedio de calificaciones entre las notas de las evaluaciones (Pre Test) antes de aplicar la metodología de Aula invertida y las notas después de someterse a la metodología (Post Test) mediante el uso TIC a la media de estudiantes que tienen la clase tradicional, a un nivel de confianza de $95 \%$.

- Ho: No existe una diferencia significativa del promedio de calificaciones entre las notas de las evaluaciones (Pre Test) antes de aplicar la metodología de Aula invertida y las notas después de someterse a la metodología (Post Test) mediante el uso TIC a la media de estudiantes que tienen la clase tradicional, a un nivel de confianza de $95 \%$.

- Prueba de hipótesis Con margen de error de $\alpha=0.05=5 \%$, tenemos que calcular la prueba $\mathrm{T}$ de Student (muestras relacionadas) y el valor de la prueba comparando las evaluaciones Pre Test (Notas) y las evaluaciones Pos Test.

\section{Conclusiones}

Se obtuvo una evaluación positiva y los resultados arrojaron que hay una diferencia significativa del promedio de calificaciones entre las notas de las evaluaciones (Pre Test) antes de aplicar la metodología del aula invertida y las notas obtenidas después de someterse a la metodología (Post Test),

Al aplicar los cuestionarios se observó que existe una diferencia significativa del promedio de calificaciones entre las notas de las evaluaciones antes de aplicar la metodología de aula invertida y las calificaciones obtenidas después de la experimentación mediante el uso TIC a la media de estudiantes que tienen la clase tradicional.

Adicionalmente se comprobó que es favorable el uso del aula invertida para un intercambio de aprendizaje bueno e interactivo ayudará utilizar el tiempo fuera del aula en realizar ciertos procesos de enseñanza-aprendizaje que se han desarrollado dentro de la misma.

Al trabajar con el aula invertida se pone de manifiesto, la importancia de las competencias que el docente tenia para insertar las TIC en el proceso de enseñanza-aprendizaje; existe abundante material educativo en la red, que puede ser útil para explicar al alumno aspectos teóricos o prácticos. 
Es importante tomar en cuenta que en todo proyecto donde se integren las TIC, además de una correcta aplicación de estrategias didácticas, es preciso que todos los actores inmersos en ello cuenten con las competencias e infraestructura necesaria para las propuestas que lleven a la innovación y al cambio, la disposición de la comunidad normalista es básica para el emprendimiento de cualquier iniciativa.

Derivando de la percepción de jóvenes respecto a la metodología y resultados de aprendizaje del curso, es interesante que, en la mayoría de los resultados de la encuesta, en una pregunta en particular arrojara $100 \%$, presumiblemente los alumnos consideraron pertinente la estrategia de aprendizaje que los videos académicos apoyarían y complementarían la explicación de las clases.

Los estudiantes normalistas actualmente están acostumbrados a buscar información en forma digital y prefieren estudiar fuera del aula tradicional. Para motivar tanto a los estudiantes más débiles, que necesitan más repetición, como a los más avanzados, que están dispuestos a tomar más responsabilidad y a determinar su propio ritmo de aprendizaje, es necesario adoptar nuevos métodos de enseñanza y aprendizaje como es el aula invertida en la cual ellos desde cualquier lugar pueden acceder a la plataforma y mirar las lecciones las veces que sea necesario.

Se logró cumplir con los objetivos determinados al inicio de la investigación porque se realizó la producción de los recursos TIC para apoyar en el proceso de enseñanza- aprendizaje de los alumnos y se identificó los factores que influyen en la no utilización de la metodología Aula invertida.

\section{Referencias}

GARCIA, David (2014). FlipClass, la propuesta 'flipped classroom' de Santillana [Consulta: 20 octubre de 2015]. Universidad de Alicante. España.

BERNAL CARRILLO, M (2014). Flipped Maths: invirtiendo la enseñanza tradicional. Máster Universitario en Formación del Profesorado de Educación Secundaria Obligatoria y Bachillerato, Formación Profesional y Enseñanzas de Idiomas. Murcia. España.

CALVILLO CASTRO, A (2014). El modelo Flipped Learning aplicado a la materia de música en el cuarto curso de Educación Secundaria Obligatoria: una investigación-acción para la mejora de la práctica docente y del rendimiento académico del alumnado. Tesis Doctoral. Valladolid: Universidad de Valladolid. España. 
GARCÍA RANGEL, M. El aula invertida y otras estrategias con uso de tic. Experiencia de aprendizaje con docentes. Universidad Interamericana para el Desarrollo. México.

CRISTIAN LADAGA, S. Clase invertida (flipped classroom) y bimodalidad: propuesta pedagógica de la cátedra taller de diseño en comunicación visual, a través de aulas web-unlp. Universidad Nacional de La Plata, Argentina.

BENITO SANCHEZ, P (2014 y 2015). Comentario de textos y The Flipped Classroom: nuevas formas de autoaprendizaje conductistaconstructivista en el aula de bachillerato. Universidad de Alicante, España.

GONZÁLEZ, B (2014). Flipped Classroom. Grandes ventajas del modelo de clase invertida. [Consultado:12 de octubre de 2015]. Badajoz, España 